\title{
Microntensile bond strenght of interface betwen dentin surface and indirect composite resin trough diferent bonding methods.
}

Guilherme dos Santos Pinto (IC), Luis Alexandre M S Paullilo (PQ)

\section{Abstract}

The aim of this study was to evaluate, in vitro, microtensile bond strength on the bond interface between indirect composite resin and dentin, trough 5 different methods of treatments on the tooth surface and restorative materials. Using the adhesive Single Bond Universal followed by Ultimate Rely $X$ cement with two variables, etch-and-rinse system to dentin and silane treatment on the indirect composite resin, in order to evaluate different techniques using the universal adhesive system in 4 of them, comparing the bonding performance to the conventional method (control-G5), which uses Scotchbond adhesive associated with the cement RelyX Arch. Bovine teeth had flattened their buccal surface until there were exposure of dentin, on which a delimited area with $25 \mathrm{~mm}^{2} .5 \times 5 \times 5 \mathrm{~mm}$ cubes made of indirect composite resin - Solidex, those were sandblasted on one of its surfaces, for 5 seconds, at a distance of $20 \mathrm{~cm}$, with Aluminum Oxide of $50 \mu \mathrm{m}$ grammage and were cemented according to manufactory's protocol on the dentin surface with different bond strategies. The samples were sectioned and the sticks specimens were obtained and submitted to microtensile test in a universal test machine(Shimatzu), the results were transformed into Mega Pascal and submitted to statistical analysis.key words: Adhesive system, Indirect restoration, adhesiveness

\section{Introduction}

One of the great challenges of restorative dentistry is the bonding effectiveness on dentin. The gold standard adhesive systems require more than one clinical step to obtain a stable adhesion to dental substrate, that can favor the occurrence of errors during it's application. Still, during cementation of prosthetic pieces, sometimes, silane is used as the surface treatment. So, the study of systems that simplify the clinical technique, by reducing clinical steps, without diminishing the quality of the adhesion are of great clinical importance.

\section{Results and Discussion}

The 40 blocks, were cemented to the buccal dentin of 40 bovine incisors according to the experimental groups $(n=8)$ : Group1:Single Bond Universal(SBU)+ phosphoricacid $37 \%(A C)+$ RelyX ceramic primer(RCP)+RelyX Ultimate $(R U)$; Group2:SBU+RCP+RU;Group3:SBU+AC+RU;

Group4:SBU+RU;Group5AC+AdperScotchbond+ $\mathrm{RCP})+$ RelyX Arch (control group). After 24 hours, those samples were sectioned and specimens in the form of sticks measuring approximately $1 \mathrm{~mm}^{2}$. The specimens were submitted to microtensile test in a universal testing machine (Shimatzu) at a rate of $0.5 \mathrm{~mm} / \mathrm{s}$ until the fracture, the results were transformed into Mega Pascal and submitted to statistical analysis. The fracture pattern was evaluated to determine the mode of failure:1- adhesive failure on dentin,2-adhesive failure on resin,3-mixed failure,4-cohesive failure within dentin,5-cohesive failure within adhesive,6cohesive failure on cement. Results: Analysis of variance(ANOVA) showed no statistically significant difference between experimental groups.Table1-Results for the microtensile strength.

\begin{tabular}{|l|l|l|}
\hline Grup & Mean(standarddeviation) & Anova \\
\hline Grup1 & $11,487(1,311)$ & $\mathrm{a}$ \\
\hline Grup2 & $14,158(5,131)$ & $\mathrm{a}$ \\
\hline Grup 3 & $16,957(3,096)$ & $\mathrm{a}$ \\
\hline Grup 4 & $17,033(4,392)$ & $\mathrm{a}$ \\
\hline Grup 5 & $16,800(9,902)$ & $\mathrm{a}$ \\
\hline
\end{tabular}

\section{Conclusions}

1- All adhesive techniques used to cement the indirect composite resin showed the same behavior in relation to tensile strength.2- The use of silane or etch-and-rinse on dentin approaches associated with SBU did not increase the bond strength values

\section{Acknowledgement}

I am thankful for the support provided by Pibic/CNPq. 